which Crimean haemorrhagic fever is a member. ${ }^{6}$ These viruses can cause severe and sometimes fatal illnesses and are distributed throughout Africa, Pakistan, and Russia.

At the time of the Johannesburg Marburg virus outbreak an extensive epizootic of mosquito-transmitted Rift Valley fever in cattle and sheep was raging in South Africa. ${ }^{7}$ Several hundred cases were diagnosed in man, particularly handlers of sick animals. Several patients died after the development of purpura, epistaxis, haematemesis, and melaena. This is believed to be the first occasion when Rift Valley fever virus has been the direct cause of fatal human infection. The outbreak was associated with abnormally heavy rains in the 1974-5 season, with a consequent increase in mosquito populations.

The dengue viruses are generally associated with a febrile illness, severe muscle and joint pains, and a rash, with few fatalities and no sequelae. In recent years a new disease pattern associated with dengue virus infection and almost exclusively confined to children between 2 and 13 years of age has emerged in south-east Asia, India, and the western Pacific. The syndrome is known as dengue haemorrhagic fever. ${ }^{8}$ It presents as a mild illness, often with upper respiratory symptoms. The mild phase lasts two to four days and is followed by a sudden collapse, with hypotension, peripheral vascular congestion, petechiae, and sometimes a rash. There are variable degrees of shock; haematemesis, melaena, and coma indicate a poor prognosis. The severe illness is thought to be due to double infection with dengue viruses with a critical interval, which may be about six months, between infection with similar viruses. The first infection probably sensitises the patient, while the second appears to produce an immunological catastrophe. The first recognised outbreak was in Manila in 1953, and 500 patients are admitted to hospital annually in the Philippines. In Bangkok and its suburbs 10000 cases were admitted between 1958 and 1965 and nearly 700 children died. ${ }^{9}$ There appears to be no decline in the incidence of the disease, which is now widespread. It tends to reappear in already affected areas. The greatest number of cases in a single year in Thailand was recorded in 1973, when 8288 cases were admitted to hospital and 310 died. ${ }^{10}$ One contributing factor may be the enormous build-up of Aedes aegypti populations in the rapidly expanding urban communities of the Orient. Haemorrhagic complications after dengue virus infection have been noted before, for instance, recorded in epidemics in Greece in 1927-8 and in Australia. A recent outbreak of dengue haemorrhagic fever occurred in a remote Pacific island without any apparent dual infection. ${ }^{11}$

The outbreaks of haemorrhagic disease in south-east Asia are always associated with large epidemics of uncomplicated febrile illnesses, usually due to dengue infection. Chikungunya virus has frequently been associated with these outbreaks, but it is not helieved to cause such a severe illness. ${ }^{912} 13$

During the last two decades another important group of viruses-the arenaviruses-has emerged. The arenaviruses include those responsible for the South American haemorrhagic fevers, the much more publicised Lassa virus, and the group prototype, lymphocytic choriomeningitis virus. Rodents appear to be the natural reservoir of infection, developing a chronic viraemia and viruria, possibly as a result of immune tolerance..$^{14}$ The viruses are believed to be transmitted to man in rodent excreta. Junin virus, the cause of Argentinian haemorrhagic fever, has caused extensive outbreaks particularly around Buenos Aires since 1953. ${ }^{15}$ Drowsiness, pallor, and facial oedema are common findings, and bleeding gums, epistaxis, haematemesis, melaena, and a petechial rash may occur. A slow pulse is characteristic, the blood pressure may drop precipitously, and oliguria and anuria ensue. Fatalities have declined because of prompt treatment. ${ }^{16}$ The Bolivian form, caused by the related Machupo virus, produced an epidemic in San Joaquin township between 1959 and 1962. In a population of 2500 there were 470 cases and 142 deaths. ${ }^{17}$ Neither Junin nor Machupo virus has spread to hospital staff attending patients in the way that Lassa fever has. This virus has caused over 100 cases, many of them secondarily infected in hospital, and over 40 deaths in three widely dispersed areas of West Africa. ${ }^{18}$

Another group of haemorrhagic fevers are those with a renal syndrome, which have been described as far east as Korea, throughout Russia, and are also found in central Europe and the Balkans. ${ }^{19}$ The syndrome was particularly prevalent among Servicemen during the Korean war. The clinical picture is of a toxic febrile illness followed by renal disease and haemorrhagic changes. Anuria and oliguria are followed by polyuria. No virus has yet been conclusively isolated from these cases.

1 Gear, J H S, unpublished data.

2 Smith, C E G, et al., Lancet, 1967, 2, 1119.

3 World Health Organisation, Weekly Epidemiological Record, 1974, 49, No 31, 261.

4 Sérié, C, et al, Bulletin of the World Health Organisation, 1968, 38, 835.

5 Boshell-M, J, American fournal of Tropical Medicine and Hygiene, 1969, $18,67$.

${ }^{6}$ Casals, J, Proceedings of the Society for Experimental Biology and Medicine, 1969, 131, 233.

7 South African Medical fournal, 1975, 49, 835.

8 World Health Organisation, Bulletin of the World Health Organisation, 1966, 35, No 1 .

${ }^{9}$ Halstead, S B, Bulletin of the World Health Organisation, 1966, 35, 3.

10 World Health Organisation, Weekly Epidemiological Record, 1974, 49, No 33, 277.

11 Barnes, W J S, and Rosen, L, American fournal of Tropical Medicine and Hygiene, 1974, 23, 495.

12 Nimmannitya, S, et al, American fournal of Tropical Medicine and Hygiene, 1969, 18, 954.

${ }^{13}$ Sarkar, J K, Chatterjee, S N, and Chakravarty, S K, Transactions of the Royal Society of Tropical Medicine and Hygiene, 1967, 61, 725.

14 Justines, G, and Johnson, K M, Nature, 1969, 222, 1090.

15 Rugiero, H R, et al, Revista de la Asociacion Medica Argentina, 1964, $78,221,281,500,611$.

16 Mettler, N E, Argentine Hemorrhagic Fever: Current Knowledge. Scientific Publication No 183. Washington, DC, Pan American Health Organisation, 1969.

17 Comisión de Investigación de la Fiebre Hemorrágica en Bolivia, Boletin de la Oficina Sanitaria Pan-Americana, 1965, 58, 93.

${ }_{18}$ Monath, T P, Tropical Doctor, 1937, 4, 155.

19 Casals, J, et al, fournal of Infectious Diseases, 1970, 122, 437.

\section{Crystal-gazing in gastroenterology}

Astrology is an unfashionable science, and there is a general scepticism about our predictive abilities. In the business and economic world estimates are today hedged about with conditions and saving clauses; in science progress is so fast, and technology so likely suddenly and unexpectedly to open whole new areas for exploration, that predictions have to be guarded. Of course there was always someone somewhere who had foreseen it all (quite frequently $\mathrm{H}$ G Wells or Leonardo da Vinci) but often he had been an isolated eccentric whose futuristic ideas were not shared by conventional authority.

The Medical Research Council is doubtless wearily aware of the hazards of scientific forecasting. Yet, unless it is to determine the distribution of its grants entirely by whim, influenced by flashes of fancy and the persuasiveness of 
individual applicants, it must try to identify areas of promise in which research satisfies the dual criteria of desirability and practicability. Its latest attempt at crystal-gazing is a report ${ }^{1}$ by a specialist subcommittee on gastroenterology, where research is burgeoning after years of neglect. Obtaining funds for gastrointestinal research is notoriously difficult-piles and bellyache generally provoke little enthusiasm among potential philanthropists. This poverty led to the establishment of the British Digestive Foundation, now slowly and with difficulty beginning to raise money, and it seems a pity that the subcommittee's first conclusion is that MRC support should continue at the present level rather than be increased. Nevertheless, the report has been published to allow comment before the MRC formulates its policy, so that gastrointestinal scientists would do well to concentrate their minds on it.

Hitherto much gastrointestinal research has been clinical rather than basic, says the subcommittee; but this is surely a reflection of the concern of British doctors with the overwhelming quantity of fascinating clinical problems and of the comparatively recent advent of measurement rather than clinical speculation in dealing with them. For this reason it was in gastroenterology that the controlled clinical trial particularly showed its early value. Gastroenterology now stands where cardiology did in 1941 when Cournand first passed his intracardiac catheter, and the MRC is right in wanting alimentary researchers to move from the bedside to the laboratory as cardiologists did 25 years ago.

The specific areas recommended for support appear to have been chosen sometimes because methods are available, sometimes because they offer hope of success, and sometimes because the clinical problem is common and serious but as yet unsolved. Critics might say that the choice of topics, with their emphasis on peptic ulceration and gastrointestinal hormones, unduly reflects the interests of the subcommittee's two Council members, Sir Andrew Watt Kay and Professor R A Gregory. But both topics satisfy all three criteria for inclusion, and if the problems of mucosal cell resistance, the role of mucus, and the causative and protective action of hormones could be elucidated mankind would be enormously benefited. (Sir Andrew may have cast a nostalgic backward glance at the comment that gastric acid secretion has had its day as a research topic.) Practical, therapeutic yields from inmunological research, another chosen sphere of activity, are likely to be slower, but its relevance to liver disease, inflammatory bowel disease, and coeliac disease is so obvious as to justify support. Fashionable fibre and its related (or not) bacteria obtain honourable mention, and a strong hint that studies on fibre need tighter control seems justified. On pancreatic disease and psychiatric aspects of gut disorders the subcommittee's inspiration weakens, and here we seem likely to remain bedfast rather than bench-bound.

Out of favour are isolated organ techniques (perhaps not if used to study cell turnover, endocrine effects, or mucus) and pressure measurements without additions such as electromyography, while controlled trials of surgery are thought best left to the NHS.

The report deals with tactics rather than strategy, though links with basic scientists and more training in laboratory techniques for young clinicians are often stressed. Long-term grants and support to basic science departments are thought desirable, but a single opaque sentence leaves the future of special MRC units unclear.

How far this report will influence the direction of gastrointestinal research is uncertain, but it is an authoritative signpost. Those actively engaged in the specialty must now comment, and public discussion is as much to be encouraged as private correspondence. Is mucus more important than acid ? Do gut electromyograms tell us more than intraluminal pressures? Is the endocrinology of the duodenum likely to be more rewarding than isclating a living organ and studying one of its functions without interference from numerous factors? The debate is open.

${ }^{1}$ Research in Gastroenterology. Report of a Medical Research Council SubCommittee 1975. London, HMSO, 1975.

\section{Systolic murmurs in the elderly}

The belief that systolic murmurs are generally benign in elderly patients has recently been challenged by Griffiths and Sheldon ${ }^{1}$ from a survey of one-third of the old people living in a particular area. Such murmurs were common, they found, the incidence rising from $33 \%$ in the age range $60-64$ to $57 \%$ in the over $85 \mathrm{~s}$; at all ages they were more frequent in women. Moreover, in $92 \%$ of the patients with a murmur this could be related to congestive cardiac failure, ischaemic heart disease, an abnormal rhythm (atrial fibrillation; supraventricular tachycardia; or heart block; but not occasional ectopic beats, which were disregarded), peripheral vascular disease, or anaemia.

Yet perhaps the original case they set out to examine had been slightly overstated. The study quoted as evidence that aortic systolic murmurs in the elderly are benign specifically excluded patients with well-recognised valvular disorders; did not use that expression "benign"; and was at pains to point out that, even if loud, such murmurs might be due to thickening of aortic valve cusps from degenerativechanges without any important degree of stenosis: an early peak to the ejection (diamond-shaped) murmur on phonocardiography supported this suggestion. ${ }^{2}$

Pathological studies indicate that the high incidence of systolic murmurs in the elderly is related to one or more abnormalities in the left heart valves. ${ }^{3}$ Griffiths and Sheldon found that aortic murmurs were more frequent than mitral murmurs ( $75 \%$ and $12 \%$, respectively). It may be difficult to differentiate aortic and mitral systolic murmurs, ${ }^{4}$ and in one series $27 \%$ of patients thought to have aortic murmurs were found to have mitral disease at necropsy. ${ }^{5}$ In the few necropsies carried out in this survey no mitral abnormalities were found in patients thought in life to have aortic murmurs. Pomerance ${ }^{6}$ has emphasised how frequent multiple cardiac abnormalities are in the elderly. In her series of 376 patients mitral ring degeneration was found in over $25 \%$ and aortic cusp disease without stenosis in over $20 \%$-while calcified aortic stenosis, rheumatic type valve disease, and bacterial or non-bacterial endocarditis were each found in only $5 \%$ or less of the patients.

The pathogenesis of aortic valve disease in the elderly differs from that found in earlier life. Postinflammatory rheumatic lesions, giving a central circular or triangular orifice, are less frequent and degenerative calcification more common. This may affect a previously bicuspid valve giving a crescentic opening (typically in men aged 65-75) or a previously normal valve giving a triradiate orifice (especially in women aged over 75$).{ }^{36}$ Presumably it was the early stages of this process which led to the conclusion that aortic systolic murmurs in the elderly may not indicate serious stenosis. ${ }^{2}$ Calcifi- 